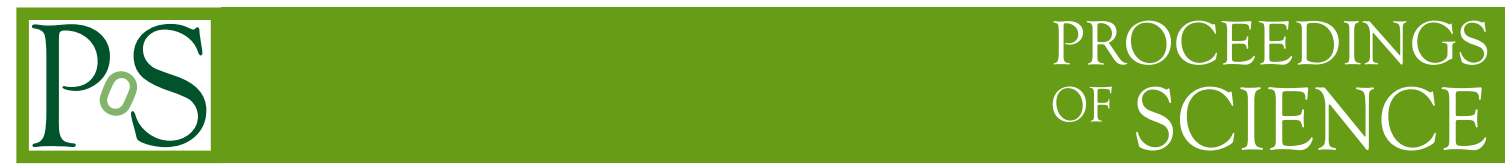

\title{
Measurements of single top quark processes with CMS
}

\section{Steffen Röcker for the CMS Collaboration}

Karlsruhe Institute of Technology (KIT), Karlsruhe, Germany

E-mail: steffen.roeckerecern.ch

\begin{abstract}
We present cross-section and properties measurements of single top quark production using data collected with the CMS detector in 2011 and 2012, at center-of-mass energies of 7 and $8 \mathrm{TeV}$. The cross sections for the electroweak production of single top quarks in the t-channel and in association with $\mathrm{W}$ bosons are measured and the results are used to place constraints on the CKM matrix element $\left|V_{t b}\right|$. Also presented is a first measurement of the $\mathrm{W}$ boson helicity fractions in single top quark event topology.
\end{abstract}

XXI International Workshop on Deep-Inelastic Scattering and Related Subjects 22-26 April, 2013

Marseilles, France 


\section{Introduction}

The standard model (SM) of particle physics allows top quark production either in pairs, via the strong interaction, or single, via the electroweak interaction. Electroweak production has many interesting properties which allow to test SM predictions or to search for new physics. The involved Wtb vertex allows the direct determination of the magnitude of the Cabbibo-Kobayashi-Maskawa (CKM) matrix element $\left|V_{t b}\right|$. Single top quark production was first observed in proton-antiproton collisions at the Tevatron at a center-of-mass energy of $\sqrt{s}=1.96 \mathrm{TeV}[1,2]$ and can now be studied in detail at the Large Hadron Collider (LHC). In the following we present cross section and properties measurements using data collected with the CMS detector [3] in 2011 and 2012 at center-of-mass energies of 7 and $8 \mathrm{TeV}$.

The virtuality of the involved $\mathrm{W}$ boson allows to differentiate between three different modes for single top quark production in the SM: $s$-channel, $t$-channel and associated $\mathrm{W}$ boson production $(\mathrm{tW})$. Exemplary Feynman diagrams for these production modes are shown in Figure 1.

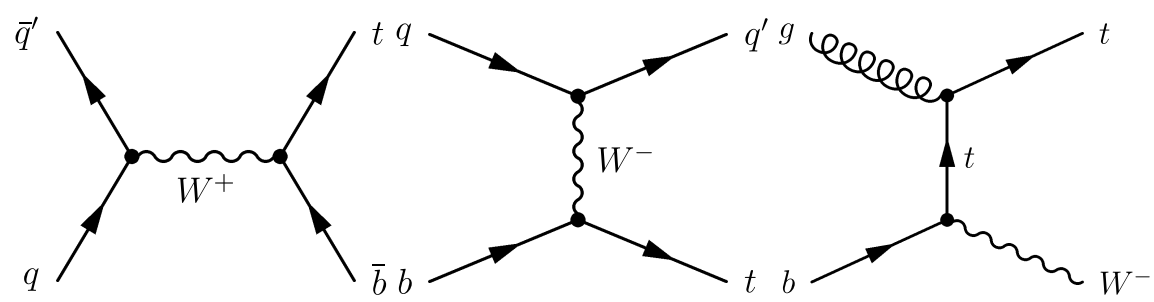

Figure 1: Exemplary Feynman diagrams for $s$-channel, $t$-channel and associated W bosons production (tW).

The cross sections for various center-of-mass energies can be found in Table 1.

Table 1: Predicted cross sections at different center-of-mass energies [4].

\begin{tabular}{|c|c|c|c|}
\hline & \multicolumn{3}{|c|}{ Predicted cross section $\sigma[\mathrm{pb}]$} \\
Collider, $\sqrt{s}$ & $s$-channel & $t$-channel & associated W production $(\mathrm{tW})$ \\
\hline$p \bar{p} \sqrt{s}=1.96 \mathrm{TeV}$ & $1.05 \pm 0.05$ & $2.1 \pm 0.1$ & $0.25 \pm 0.03$ \\
\hline$p p \sqrt{s}=7 \mathrm{TeV}$ & $4.6 \pm 0.2$ & $64.6_{-1.9}^{+2.6}$ & $15.7 \pm 1.2$ \\
\hline$p p \sqrt{s}=8 \mathrm{TeV}$ & $5.6 \pm 0.2$ & $87.2_{-2.4}^{+3.4}$ & $22.2 \pm 0.8$ \\
\hline
\end{tabular}

All signal samples used in the following analyses have been produced with the NLO Monte Carlo (MC) event generator POWHEG [5] and hadronized with PYTHIA [6].

\section{Single top quark $t$-channel measurements}

The event selection for $t$-channel measurements, where the top quark decays to a $\mathrm{b}$ quark and a leptonically decaying $\mathrm{W}$ boson, requires one isolated charged lepton (muon or electron). Events with additional softer leptons are vetoed. Exactly two jets, which can be very forward $(|\eta|<4.5)$, are required, whereas one jet has to be b-tagged. In order to reduce the background from QCD multijet production a cut on the transverse mass of the $\mathrm{W}$ boson, $M_{\mathrm{T}}(\mathrm{W})$, or missing transverse energy, MET, is applied for muons and electrons, respectively. 
After the event selection, the main background processes left are $\mathrm{W}$ boson plus jets production and top quark pair production. Since the small remaining QCD multijet background is difficult to model, and the number of remaining MC events after the event selection is very small, this background process is estimated from data by a fit to $M_{\mathrm{T}}(\mathrm{W})$ for muons, or MET for electrons, in an orthogonal data set with inverted isolation criteria for muons, or inverted electron ID criteria for electrons.

\subsection{Cross section measurement}

The $t$-channel cross section has been measured by the CMS Collaboration at $7 \mathrm{TeV}$ [7] with a data set of $1.17 \mathrm{fb}^{-1}$ for muons and $1.56 \mathrm{fb}^{-1}$ for electrons. Three different analyses have been performed at $7 \mathrm{TeV}$. One analysis uses a robust template fit to exploit the forward light quark pseudorapidity $\left(\left|\eta_{j^{\prime}}\right|\right)$ and a data driven estimation of the important backgrounds in a top quark mass sideband. This analysis has also been used for a measurement at $8 \mathrm{TeV}$ with an integrated luminosity of $5 \mathrm{fb}^{-1}$ [8] where additional cuts where necessary due to the higher center-of-mass energy and increased pile-up.

In addition to the robust estimate with $\left|\eta_{j^{\prime}}\right|$, two multivariate analyses (MVA), that cross check each other, have been performed at $7 \mathrm{TeV}$. Both MVAs, boosted decision tree (BDT) and artificial neural network $(\mathrm{NN}$,$) use several well described input variables, including the light quark pseudo-$ rapidity, that have been checked in control regions. Multiple jet and tag categories, with up to 4 jets and equal to or more than two b-tagged jets, are utilized. The output discriminators of both MVAs in the signal region are shown in Figure 2.
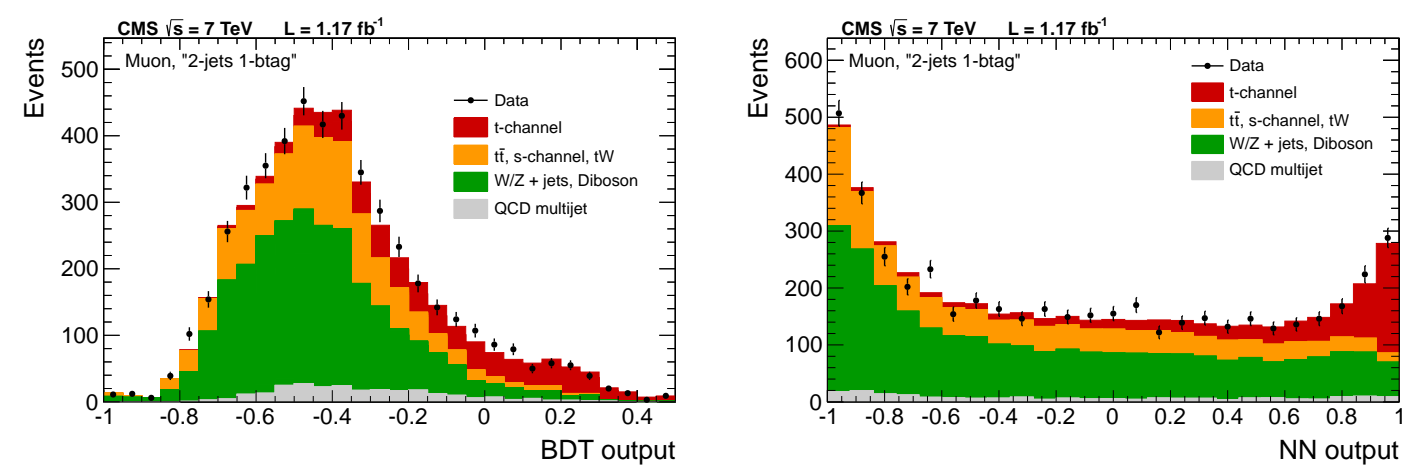

Figure 2: Output of multivariate discriminators in the signal region used for the $7 \mathrm{TeV} t$-channel cross section measurement.

Both MVAs utilize a Bayesian method for the statistical inference where experimental uncertainties are marginalized as nuisance parameters and theoretical uncertainties are estimated with pseudo experiments and a likelihood fit. The correlation of all three analyses has been estimated with pseudo experiments. Combining all three measurements with the BLUE method [9] yields a cross section of

$$
\sigma_{\mathrm{t}-\mathrm{ch} .}=67.2 \pm 3.7 \text { (stat.) } \pm 3.0 \text { (syst.) } \pm 3.5 \text { (theor.) } \pm 1.5 \text { (lum.) } \mathrm{pb}
$$

The analysis at $8 \mathrm{TeV}$ measures a cross section of 


$$
\sigma_{\mathrm{t}-\mathrm{ch} .}=80.1 \pm 5.7 \text { (stat.) } \pm 11.0 \text { (syst.) } \pm 4.0 \text { (lum.) pb }
$$

Both results are well compatible with the SM predictions presented in Table 1. The CKM matrix element $\left|V_{t b}\right|$ can be extracted from this cross sections assuming $\left|V_{t b}\right|^{2} \gg\left|V_{t d}\right|^{2}+\left|V_{t s}\right|^{2}$ by taking the square root of the fraction of measured and predicted cross section $\left|V_{t b}\right|=\sqrt{\frac{\sigma_{t-c h}}{\sigma_{t-c h}^{t h}}}$. The extracted value for $7 \mathrm{TeV}$ is

$$
\left|f_{L_{V}} V_{t b}\right|=1.020 \pm 0.046 \text { (exp.) } \pm 0.017 \text { (theor.) }
$$

and the result for $8 \mathrm{TeV}$ is

$$
\left|f_{L_{V}} V_{t b}\right|=0.96 \pm 0.08 \text { (exp.) } \pm 0.02 \text { (theor.), }
$$

where $f_{L_{V}}$ is a possible anomalous form factor for a left-handed vector coupling from beyond standard model (BSM) contributions, e.g. vector-like quarks. Setting $f_{L_{V}}$ to 1 and constraining $\left|V_{t b}\right|$ to the interval $[0,1]$ yields a confidence interval of

$$
0.92<\left|V_{t b}\right| \leq 1 @ 95 \% \text { C.L. }
$$

for the $7 \mathrm{TeV}$ measurement by employing a unified Feldman-Cousins approach. Applying the same method for the results measured at $8 \mathrm{TeV}$ yields a confidence interval of

$$
0.81<\left|V_{t b}\right| \leq 1 @ 95 \% \text { C.L }
$$

\subsection{Charge asymmetry}

Since the LHC is a proton-proton collider and the top quark charge is connected to the incoming light quark charge in the $t$-channel, more top than anti-top quarks are produced. The cross section ratio $R_{t}=\frac{\sigma_{t}}{\sigma_{\bar{t}}}$ depends on the $\mathrm{u}(\mathrm{d})$ quark parton densitiy function (PDF), it is however also sensitive to new physics.

CMS has measured the $t$-channel charge asymmetry at $8 \mathrm{TeV} \mathrm{[10]} \mathrm{with} \mathrm{an} \mathrm{integrated} \mathrm{luminos-}$ ity of $12.2 \mathrm{fb}^{-1}$. The analysis utilizes the same method as the $8 \mathrm{TeV}$ cross section measurement, performing a template fit to $\left|\eta_{j^{\prime}}\right|$ separately for positive and negative charged leptons. Systematic uncertainties have been estimated with pseudo experiments. Due to taking the ratio the luminosity uncertainty cancels out and jet energy scale (JES), jet energy resolution (JER) and missing transverse energy uncertainties are reduced. The resulting cross sections and the ratio for top and anti-top production in the $t$-channel are

$$
\begin{aligned}
\sigma_{\mathrm{t}-\text { ch.top }} & =49.9 \pm 1.9 \text { (stat. }) \pm 8.9(\text { syst. }) p b \\
\sigma_{\mathrm{t}-\text { ch.,anti-top }} & =28.3 \pm 2.4(\text { stat. }) \pm 4.9(\text { syst. }) p b \\
R_{\mathrm{t}-\text { ch. }} & =1.76 \pm 0.15 \text { (stat. }) \pm 0.22 \text { (syst. })
\end{aligned}
$$

The result and predictions for different PDFs are shown in Figure 3, the result is compatible with SM predictions. With more data and higher precision, the charge ratio in the $t$-channel could be used to constrain $u(d)$ quark PDFs. 


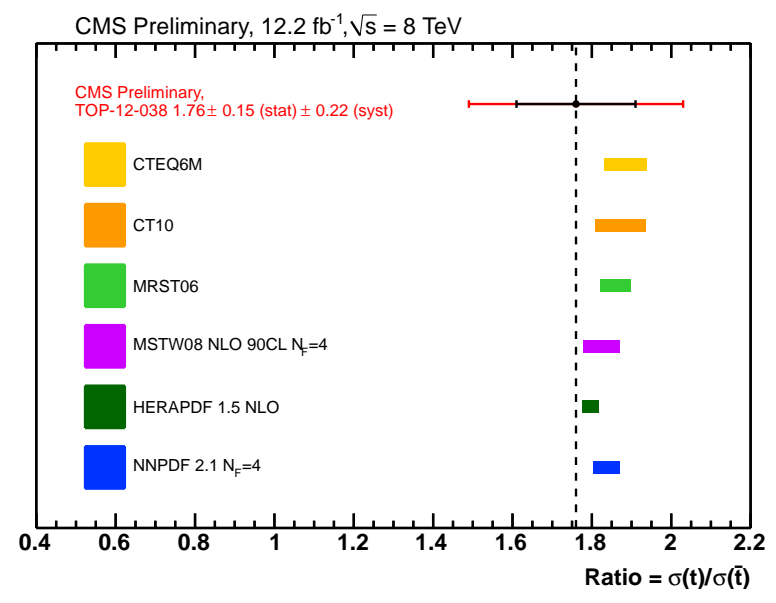

Figure 3: Comparison of the measured cross section ratio $R_{\mathrm{t}-\mathrm{ch}}$. with the prediction obtained using different PDF sets. Error bars for the different PDF sets include the statistical uncertainty, the uncertainty on the factorization and renormalization scales and the uncertainty on the top quark mass.

\subsection{W boson helicity fractions}

The $\mathrm{W}$ boson helicity fractions measurement in single top event topology [11] uses the same selections as the 7 and $8 \mathrm{TeV}$ cross section measurements with muons.

The fact that $\mathrm{W}$ bosons from top quark decays are polarized enables the measurement of the $\mathrm{W}$ boson helicity fractions using the variable $\cos \theta_{l}^{*}$, where $\theta_{l}^{*}$ is the angle between the charged lepton 3-momentum in the $\mathrm{W}$ boson rest frame and the $\mathrm{W}$ boson 3-momentum, in the top-quark rest frame. The angular decay distribution is given by

$$
\frac{1}{\Gamma} \frac{d \Gamma}{d \cos \theta_{l}^{*}}=\frac{3}{8}\left(1-\cos \theta_{l}^{*}\right)^{2} F_{L}+\frac{3}{8}\left(1+\cos \theta_{l}^{*}\right)^{2} F_{R}+\frac{3}{4} \sin ^{2} \theta_{l}^{*} F_{0} .
$$

The SM predicts helicity fractions of $F_{L} \approx 0.30, F_{R} \approx 0$ and $F_{0} \approx 0.70$. The helicity fractions can differ from SM predictions through modifications of the Wtb vertex by BSM contributions. A general Lagrangian of the Wtb vertex in effective field theory can be written with a dimension- 6 operator

$$
\mathscr{L}_{W t b}^{\text {anom. }}=-\frac{g}{\sqrt{2}} \bar{b} \gamma^{\mu}\left(V_{L} P_{L}+V_{R} P_{R}\right) t W_{\mu}^{-}-\frac{g}{\sqrt{2}} \bar{b} \frac{i \sigma^{\mu v} q_{v}}{m_{W}}\left(g_{L} P_{L}+g_{R} P_{R}\right) t W_{\mu}^{-}+\text {h.c. }
$$

In the SM, only a left-handed vector coupling equal to $V_{t b}$ is expected and the right-handed vector coupling and all tensor couplings are zero, $V_{L}=V_{t b} \approx 1, V_{R}=g_{L}=g_{R}=0$.

For the measurement, all events in processes with a Wtb vertex are reweighted and the measurement is performed with likelihood fit to the distribution of $\cos \theta_{l}^{*}$, shown in Figure 4. The result for the combined measurement with a combined likelihood at 7 and $8 \mathrm{TeV}$ is

$$
\begin{array}{r}
\left.\left.F_{L}=0.293 \pm 0.069 \text { (stat. }\right) \pm 0.030 \text { (syst. }\right) \\
F_{0}=0.713 \pm 0.114(\text { stat. }) \pm 0.023(\text { syst. }) \\
\left.\left.F_{R}=-0.006 \pm 0.057 \text { (stat. }\right) \pm 0.027 \text { (syst. }\right)
\end{array}
$$



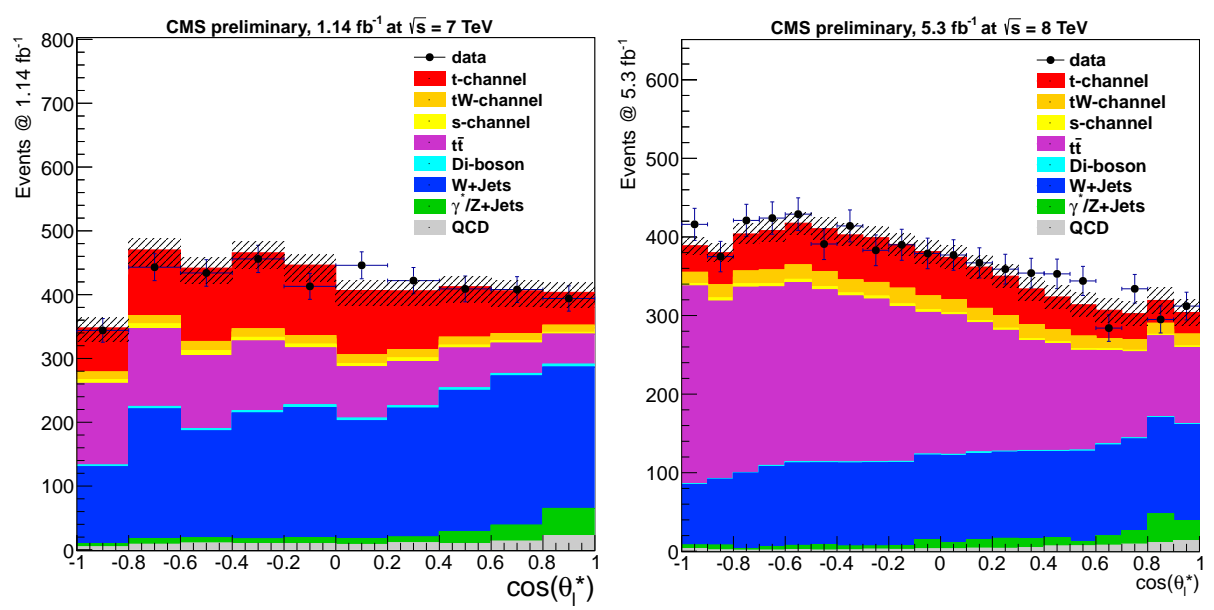

Figure 4: Distribution of $\cos \theta_{l}^{*}$ for 7 (left) and $8 \mathrm{TeV}$ (right).

The result is consistent with SM V-A couplings and $t \bar{t}$ measurements from the LHC combination [12]. More data is needed for tighter constraints.

\section{Associated W boson production}

A search for associated $\mathrm{W}$ boson production has been performed by the CMS collaboration with a data set of $4.9 \mathrm{fb}^{-1}$ at a center-of-mass energy of $7 \mathrm{TeV}$ [13].

The selection for dileptonic final states requires two isolated leptons $(e e, e \mu, \mu \mu)$ with opposite charge and missing transverse energy stemming from neutrinos. The signal region consists of events with one jet which has to be b-tagged. Events with two jets, where one or two jets have to be $\mathrm{b}$-tagged, are used to constrain $t \bar{t}$. Events where the dilepton mass is in the $\mathrm{Z}$ boson mass window are excluded. Important backgrounds remaining after the selection are $t \bar{t}$ and $Z / \gamma^{*}$ plus jets. Two independent analysis have been performed, a simple cut based analysis and a MVA with a BDT. The result has been obtaind with the BDT analysis and cross checked with the cut based analysis, both results are consistent.

In order to separate $t \bar{t}$ and $\mathrm{tW}$ the BDT is trained with four kinematical variables: the transverse momentum of the leading jet, the transverse momentum of the system, the scalar sum of the momenta of the system and the difference in angular separation between the direction of MET and the closest lepton. The output of the BDT in the signal region for data and MC is shown in Figure 5.

The measurement is performed by fitting the BDT output in the signal and $t \bar{t}$ control regions for all lepton categories simultaneously. With an observed significance of $4 \sigma$ (expected significance: $3.6_{-0.9}^{+0.8} \sigma$ ) first evidence for associated $\mathrm{W}$ boson production has been obtained. The measured cross section is

$$
\sigma_{\mathrm{tW}}=16_{-4}^{+5} \mathrm{pb}
$$

which can be translated into a value for the CKM matrix element $\left|V_{t b}\right|$

$$
\left|V_{t b}\right|=1.01_{-0.13}^{+0.16}(\text { exp. })_{-0.04}^{+0.03}(\text { th. })
$$




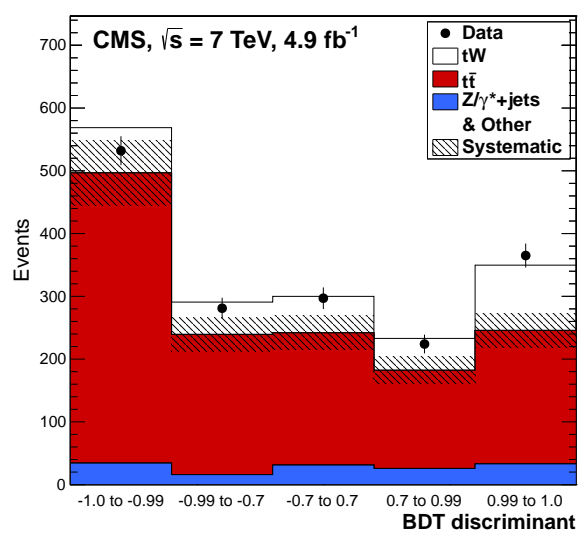

Figure 5: BDT discriminator output in the signal region.

Constraining $\left|V_{t b}\right|$ to SM values results in a confidence interval of

$$
0.78 \leq\left|V_{t b}\right| \leq 1.0 @ 90 \% \text { C.L. }
$$

The measured cross section is consistent with the SM prediction.

\section{Conclusions}

CMS has measured the $t$-channel cross sections at center-of-mass energies of 7 and $8 \mathrm{TeV}$. The very precise result with $7 \mathrm{TeV}$ data allows the up to now most precise direct constraint on the CKM matrix element $\left|V_{t b}\right|$, which is well compatible with the SM prediction. The charge ratio in the $t$-channel has been measured at $8 \mathrm{TeV}$ and is well compatible with different predictions. First evidence for associated $\mathrm{W}$ boson production has been found with $7 \mathrm{TeV}$ data, the next step is the definitive establishment of this production mode. For the first time, $\mathrm{W}$ boson helicity fractions have been measured in single-top event topology. In the future, this measurement can be added to the LHC wide combination of this property of top quark decay.

The analyses currently published by the CMS Collaboration have been using only half of the data which is available now. With more data, detailed studies of electroweak top quark production and interesting property measurements will be possible.

\section{References}

[1] CDF Collaboration, Phys. Rev. D82, 112005 (2010)

[2] D0 Collaboration, Phys. Rev. D84, 112001 (2010)

[3] CMS Collaboration, JINST 03, S08004 (2008)

[4] N. Kidonakis, Phys. Rev. D81, 054028; Phys. Rev. D83, 091503 (R) and Phys. Rev. D82, 054018

[5] E. Re, Eur. Phys. J. C 71, 1547 (2011); S. Alioli, P.Nason, C. Oleari et al., JHEP 06, 043 (2010); S. Alioli, P. Nason, C. Oleari et al., JHEP 09 , 111 (2009); S. Frixione, P. Nason, and C. Oleari, JHEP 11, 070 (2007) 
[6] T. Sjöstrand, S. Mrenna, and P. Z. Skands, JHEP 05, 026 (2006)

[7] CMS Collaboration, JHEP 12, 035 (2012)

[8] CMS Collaboration, CMS PAS TOP-12-011 (2012)

[9] L. Lyons, D. Gibaut and P. Clifford, Nucl. Instrum. Meth. A 270, 110 (1988)

[10] CMS Collaboration, CMS PAS TOP-12-038 (2012)

[11] CMS Collaboration, CMS PAS TOP-12-020 (2012)

[12] CMS Collaboration, CMS PAS TOP-12-025 (2012)

[13] CMS Collaboration, Phys. Rev. Lett. 110, 022003 (2013) 